\title{
Inhibition of angiopoietin 2 attenuates lumen formation of tumour-associated vessels in vivo
}

\author{
REI SUZUKI $^{1}$, HIROFUMI YAMAMOTO ${ }^{1}$, CHEW YEE NGAN $^{1,2,3}$, MASAHISA OHTSUKA $^{1}$, KOTARO KITANI $^{1}$, \\ MAMORU UEMURA ${ }^{1}$, JUNICHI NISHIMURA ${ }^{1}$, ICHIRO TAKEMASA ${ }^{1}$, TSUNEKAZU MIZUSHIMA ${ }^{1}$, \\ MITSUGU SEKIMOTO ${ }^{1}$, TOSHINARI MINAMOTO ${ }^{2}$, YUICHIRO DOKI ${ }^{1}$ and MASAKI MORI ${ }^{1}$ \\ ${ }^{1}$ Department of Surgery, Gastroenterological Surgery, Graduate School of Medicine, Osaka University, \\ Suita, Osaka; ${ }^{2}$ Division of Translational and Clinical Oncology, Cancer Research Institute, \\ Kanazawa University, Kanazawa, Ishikawa, Japan
}

Received April 29, 2013; Accepted June 14, 2013

DOI: 10.3892/ijo.2013.2076

\begin{abstract}
Anti-angiogenic therapy, inhibition of a co-operative process with vascular endothelial cells and pericytes could be an effective strategy to treat malignant tumours. Apart from vascular endothelial growth factor (VEGF), angiopoietin 2 (Ang2) is a promising target of anti-angiogenic therapy. Although inhibition of Ang2 has been shown to decrease tumour size in preclinical and phase I trials, its mechanisms of action remain largely unknown. To elucidate the mechanisms of Ang2 inhibition, we have focused on differentiation of the vessels as well as on growth of the vessels, especially in vivo. L1-10, a selective Ang2 inhibitor was used. The in vitro effects of Ang2 inhibition or addition of Ang2 using HUVECs were also examined. Growth and differentiation of tumour-associated vessels were investigated in xenografts derived from a colon cancer treated by L1-10. Effects of VEGF inhibition were also examined to discriminate Ang2-specific action on the tumour-associated vessels. In vitro studies showed that VEGF enhanced proliferation and tube formation of HUVECs, and caused a significant increase in Rac1 and CDC42 expression when cultured in the collagen matrix gel, whereas neither Ang2 nor L1-10 affected in vitro behaviour of HUVECs or levels of the proteins. In vivo, on the other hand, we found that Ang2
\end{abstract}

Correspondence to: Dr Hirofumi Yamamoto, Department of Surgery, Gastroenterological Surgery, Graduate School of Medicine, Osaka University, 2-2 Yamadaoka, Suita, Osaka 565-0871, Japan E-mail: hyamamoto@gesurg.med.osaka-u.ac.jp

Present address: ${ }^{3}$ Joint Genome Institute, Walnut Creek, CA, USA

Abbreviations: Ang2, angiopoietin 2; CRC, colorectal cancer; EC, endothelial cell; VEGF, vascular endothelial growth factor; PBS, phosphate-buffered saline; RT-PCR, reverse transcription polymerase chain reaction

Key words: angiopoietin 2, vascular differentiation, lumen formation, vascular endothelial growth factor inhibition with treatment of L1-10 dose-dependently decreased tumour growth. Furthermore, we found that L1-10 treatment extends the tumour-associated vessels whilst it suppressed a sound lumen formation. Histological analysis on xenografts suggests that Ang2 inhibition could have disturbed in vivo vascular differentiation. Our data provide a novel aspect that Ang2 may play an essential role in in vivo vascular differentiation, thus supporting a rationale for Ang2-targeted therapy against colon cancer.

\section{Introduction}

Angiogenesis, a co-operative process with vascular endothelial cells (ECs) and pericytes, is essential for tumour growth and expansion because the blood vessels supply malignant cells with sufficient oxygen and nutrition. Interruption of this process, therefore, could be an effective strategy for preventing malignant tumours. The putative angiogenic factor vascular endothelial growth factor (VEGF) is the best known to be involved in the growth and development of colorectal cancer (CRC) and its hepatic metastases (1-3). Anti-VEGF monoclonal antibody, bevacizumab (BV) is already clinically feasible in combination with conventional chemotherapies as the first anti-angiogenic drug that is proven to bring better prognosis of patients with colorectal cancer (CRC) in a phase III randomized controlled trial (4).

Besides VEGF, other important endothelial growth factors are angiopoietins (Angs), which are ligands for the endothelium-specific tyrosine kinase receptor Tie2 (5). Angs play a role in normal vascular development and in embryonic angiogenesis. Among 4 subtypes (Ang1, 2, 3 and 4), the best-characterized are Ang1 and its natural antagonist, Ang2. Ang1 is widely expressed in normal adult tissues, while Ang 2 is expressed primarily at sites of vascular remodeling, such as the ovaries, uterus and placenta (5). Angiogenesis requires migration and remodeling of ECs derived from pre-existing blood vessels and regulation of the perivascular microenvironment. Thus, Ang2 destabilizes pre-existing vessels by weakening interactions between ECs and periendothelial supporting cells (PESCs) (3), also called vascular pericytes. Ang1 subsequently acts, via the Tie 2 receptor, to remodel the primitive vessels and to help 
maintain and stabilize mature vessels (6). Recent preclinical studies have shown that angiopoietin 2 may be another promising target against colon cancer through inhibition of either Ang2 (7-9) or Ang2+Ang1 (9,10).

There are two aspects of angiogenesis, i.e. growth and differentiation of the vessels. Although vascular growth (EC proliferation) has been examined by estimating vessel count and vessel size so far, little is known about vascular differentiation, i.e., vacuole or lumen formation, especially in vivo. In the present study, we investigated the effects of Ang2 on vascular growth and differentiation, in vitro and in vivo. We first examined in vitro effects of Ang2 inhibition or addition of Ang2 using HUVECs. Secondly we examined growth and differentiation of tumour-associated vessels when xenografts derived from a colon cancer were treated by the Ang2 inhibitor L1-10. Effect of VEGF inhibition was also examined to discriminate Ang2 specific action on the tumour-associated vessels. Our data provide a novel aspect that Ang2 may play an essential role in in vivo vascular differentiation, and therefore support a rationale for Ang2-targeted therapy against colon cancer.

\section{Materials and methods}

Cell lines. Human umbilical vascular endothelial cell (HUVEC) was purchased from Takara Bio Co. (Shiga, Japan). Human colon cancer cells (HCT116, DLD1, SW480) were purchased from the American Type Culture Collection (Manassas, VA). KM12SM (11) was a kind gift from Professor T. Minamoto (Cancer Research Institute, Kanazawa University, Kanazawa, Japan). Colon cancer cells were grown in DMEM supplemented with $10 \%$ fetal bovine serum (FBS), $100 \mathrm{U} / \mathrm{ml}$ penicillin, and $100 \mu \mathrm{g} / \mathrm{ml}$ streptomycin in $5 \% \mathrm{CO}_{2}$ at $37^{\circ} \mathrm{C}$. HUVEC were grown on MCDB131 culture medium (Chlorella Inc., Tokyo, Japan) supplemented with $10 \% \mathrm{FBS}$, antibiotics, and $10 \mathrm{ng} / \mathrm{ml}$ basic fibroblast growth factor.

Attached collagen gel culture. HUVECs $\left(2 \times 10^{6}\right.$ cells $\left./ \mathrm{ml}\right)$ were cultured on $0.03 \%$ type I collagen (Cellmatrix I-A, Nitta Gelatin Inc., Osaka, Japan) coated dishes. Collagen solution $(0.3 \%$ ) was diluted by Medium 199 (Life Technologies, Carlsbad, CA) and reconstruction buffer $(50 \mathrm{mM} \mathrm{NaOH}$, $260 \mathrm{mM} \mathrm{NaHCO}, 200 \mathrm{mM}$ HEPES, according to the Nitta Gelatin manual). After collagen coating, cell suspension was seeded, then medium containing appropriate concentration of reagent such as VEGF, Ang2 and L1-10 was added, and $24 \mathrm{~h}$ later, same volume of PBS, 1/25 volume of Collagenase N-2 (Nitta Gelatin Inc.) solution were added into the well, incubated at $37^{\circ} \mathrm{C}$ with mild shaking. Suspension was spun down, pellet was resuspended with same volume of PBS and 1/50 volume of Collagenase $\mathrm{N}-2$. After $10 \mathrm{~min}$ incubation at $37^{\circ} \mathrm{C}$, pellet was lysed and supernatant was used for western blot analysis (12).

Collagen gel matrix culture. In vitro formation of tubular structures by HUVEC was examined using collagen gel matrix culture. Collagen gel $(0.06 \%)$ layer was made (base layer). Collagen gel $(0.06 \%)$ suspended with HUVECs was added onto base layer, and immediately polymerized at $37^{\circ} \mathrm{C}$. Medium containing appropriate concentration of reagent such as VEGF, Ang2 or L1-10 was added. Twenty-four or $48 \mathrm{~h}$ later,
HUVECs were harvested as mentioned above or observed under an inverted microscope. Cell concentration of HUVECs for western blot analysis and morphogenesis were $1 \times 10^{6}$ cells/ $\mathrm{ml}$ and $3 \times 10^{6}$ cells $/ \mathrm{ml}$, respectively.

Reagents and antibodies. Human recombinant VEGF and mouse IgG was obtained from IBL Co. Ltd. (Gunma, Japan). Ang2 was purchased from (R\&D Systems, Minneapolis, MN). The following antibodies were used at appropriate concentrations as recommended by the manufacturers: antibodies for angiopoietin 2 (C-19, Santa Cruz Biotechnology, Santa Cruz, CA), VEGF (A20, Santa Cruz Biotechnology), Tie2 (H-176 for immunoprecipitation, C-20 for western blot analysis, Santa Cruz Biotechnology), phosphorylated Tie2 (Tyr992,\#4221, Cell Signaling Technology, Danvers, MA), actin (Sigma-Aldrich, St. Louis, MO), Rac1 (Cytoskeleton, Denver, CO), CDC42 (BD Biosciences, San Jose, CA), CD31 and $\alpha$ smooth muscle actin (Dako, Glostrup, Denmark).

L1-10. L1-10, an Ang2 neutralizing peptibody (geneticallyengineered peptide-Fc fusion protein) was kindly donated by Amgen Inc. (Seattle, WA). L1-10 is a specific inhibitor of angiopoietin-2, and inhibits interactions between Tie2 in endothelial cells and human or mouse angiopoietin-2 $(7,13,14)$.

Binding activity of L1-10 to Ang2 was measured by ELISA. Recombinant human angiopoietin-2 (R\&D Systems) was immobilized on a plate. After blocking with $1 \%$ BSA, 1 pM recombinant human Tie2/Fc Chimera (R\&D Systems) was added, from 500 to $0.02 \mathrm{nM}$ of L1-10 and recombinant human IgG1 Fc (R\&D Systems). Molecular weight of L1-10 was assumed as $62.5 \mathrm{kDa}$ and that of IgG Fc was $26.6 \mathrm{kDa}$. Anti-Tie2 monoclonal antibody (BD Biosciences) at $0.25 \mu \mathrm{g}$ / $\mathrm{ml}$ was added. After washing, $0.05 \mu \mathrm{g} / \mathrm{ml}$ anti-mouse IgG (Goat IgG) Fab' conjugated with HRP (IBL Co. Ltd.) was added. Color development was done by incubating with tetramethyl benzidine solution for $30 \mathrm{~min}$. Absorbance at $450 \mathrm{~nm}$ was measured by microplate reader.

Western blot analysis. Western blot analysis was performed as we described previously (12). Briefly, the protein samples $(25 \mu \mathrm{g})$ were separated by 10 or $12.5 \%$ PAGE followed by electroblotting onto a polyvinylidene difluoride (PVDF) membrane. The membrane was incubated with the primary antibodies at the appropriate concentrations (1:100 for Ang2, 1:200 for VEGF and Tie2, 1:250 for CDC42 and 1:1,000 for Rac1 actin and phosphorylated Tie2). The protein bands were detected using the Amersham enhanced chemiluminescence detection system (GE Healthcare, Buckinghamshire, UK).

To detect phosphorylated Tie2, lysates of HUVEC were immunoprecipitated with an anti-Tie 2 antibody (H-176, Santa Cruz Biotechnology). Immunocomplexes were recovered on Protein A-Sepharose (GE Healthcare) and separated by SDS-PAGE, transferred to blotting membrane as described above, then probed with anti-phosphorylated Tie 2 antibody (\#4221, Cell Signaling Technology).

Measurement of Ang2 and VEGF secretion in culture medium. Each cell line was cultured until about $70 \%$ confluence in DMEM supplemented with FBS. The medium was then 
Table I. Administration schedules of combination treatment.

\begin{tabular}{lccccc}
\hline & & & & \multicolumn{2}{c}{ Dose } \\
\cline { 5 - 6 } Group & Drug & Route & Interval (days) & Early & Late \\
\hline Control & IgG & i.p. & 3 & $200 \mu \mathrm{g}$ & $150 \mu \mathrm{g}$ \\
VEGF & Anti-VEGF antibody & i.p. & 3 & $200 \mu \mathrm{g}$ & $150 \mu \mathrm{g}$ \\
Combination & Anti-VEGF antibody & i.p. & 3 & $200 \mu \mathrm{g}$ & $150 \mu \mathrm{g}$ \\
& L1-10 & s.c. & 2 & $5 \mathrm{mg} / \mathrm{kg}$ & $10 \mathrm{mg} / \mathrm{kg}$ \\
\hline
\end{tabular}

replaced with new medium without FBS, collected $24 \mathrm{~h}$ later, and stored at $-80^{\circ} \mathrm{C}$. Ang2 and VEGF levels were analyzed using the QuantikineHuman Angiopoietin-2 immunoassay kit (R\&D Systems) and the Human VEGF assay kit (IBL Co. Ltd.), respectively.

Animals. Female 4-week-old athymic nude mice were purchased from Nihon CREA Inc. (Tokyo, Japan) and were housed under pathogen-free conditions in microisolator cages with irradiated rodent chow and water available ad libitum. The experimental protocol was approved by the Ethics Review Committee for Animal Experimentation of Osaka University School of Medicine.

Subcutaneous xenograft model. The most actively secreting VEGF and Ang2 cell line was KM12SM. KM12SM cells at $80-90 \%$ confluence was used in experiments. A total of $1 \times 10^{6}$ cells $\left(5 \times 10^{6}\right.$ cells $/ 0.1 \mathrm{ml}$ DMEM without FBS) was subcutaneously inoculated in the right flank of each mouse. The doses of each drug were based on the results of preliminary experiments. Mice were randomly assigned to the groups.

Single agent treatment: L1-10 or anti-VEGF antibody treatment was started immediately after inoculation. There were four mice in each group. L1-10 was administered subcutaneously into the left flank skin at $2 \mathrm{mg} / \mathrm{kg}, 5 \mathrm{mg} / \mathrm{kg}$ every two days. Anti-VEGF antibody (200 $\mu \mathrm{g}$, IBL Co. Ltd.), was administered intraperitoneally every three days. Control groups for each drug were administered mouse immunoglobulin $(\operatorname{IgG})$ in the same manner as the experimental group. Treatment was continued for 18 days. Mice were sacrificed at day 20, and tumours were harvested for histological examinations.

Combination treatment with early administration: L1-10 and anti-VEGF antibody injections were initiated immediately after inoculation. There were five mice in each group. Anti-VEGF antibody $(200 \mu \mathrm{g})$ was administered intraperitoneally every three days. Combination group was administered $5 \mathrm{mg} / \mathrm{kg}$ of L1-10 subcutaneously every two days and $200 \mu \mathrm{g}$ of anti-VEGF antibody was administered intraperitoneally every three days. Control group was administered $200 \mu \mathrm{g}$ of mouse IgG intraperitoneally every three days. Treatment was continued to day 18 . Mice were sacrificed at day 20 , and tumours were harvested for histological examinations.

Combination treatment with late administration: L1-10 and anti-VEGF antibody treatment were initiated 5 days after inoculation. There were four mice in each group. Dose was $10 \mathrm{mg} / \mathrm{kg}$ for L1-10, $150 \mu \mathrm{g}$ for anti-VEGF antibody, $150 \mu \mathrm{g}$ for IgG, and combination (L1-10 and anti-VEGF antibody) treatment was applied. Treatment was continued to day 31 . At day 34 mice were sacrificed.

After inoculation of KM12SM cells into nude mice, control $\mathrm{IgG}$, anti-VEGF antibody and L1-10 were administered as summarized in Table I.

Evaluation of antitumour activity. Tumour size and body weight were measured every two days. Tumour size was measured by an electronic caliper. Tumour volume was calculated according to the following formula: length $(\mathrm{mm}) \mathrm{x}$ width $^{2}(\mathrm{~mm}) / 2$. Mice were sacrificed at the final day of experiment. Gross autopsy findings were noted.

Immunohistochemistry. Immunostaining was done as described previously (3). Briefly, after deparaffinization, heat antigen retrieval was done in $10 \mathrm{mM}$ citrate buffer $(\mathrm{pH} \mathrm{6.0)}$ at $95^{\circ} \mathrm{C}$ for $40 \mathrm{~min}$. The slides were then processed for immunohistochemistry using the Vectastain Elite avidin-biotin complex kit (Vector Laboratories, Burlingame, CA). Primary antibodies were applied to sections at a dilution of 1:750 for $\mathrm{CD} 31$ and incubated overnight at $4^{\circ} \mathrm{C}$. For the negative control, non-immunized immunoglobulin $\mathrm{G}$ (Vector Laboratories) was used as a substitute for the primary antibody.

Double-staining of endothelial cells and pericytes was performed with anti-CD31 antibody and anti- $\alpha$-SMA antibody, respectively. First, CD31 staining which yields a brown color was performed. After removal of the CD31 antibody by thorough washing in $0.1 \mathrm{M}$ glycine solution ( $\mathrm{pH} 2.2$ ) for $1 \mathrm{~h}$, mouse monoclonal anti-human SMA antibody at a dilution of 1:200 was applied to the section for $2 \mathrm{~h}$ at room temperature. This step was followed by incubation with anti-mouse secondary antibody conjugated with a dextran backbone containing alkaline phosphatase (EnVision AP; Dako) for 30 min. Color development (deep pink) based on alkaline phosphatase activity was achieved using fuchsin solution.

Image analysis. CD31-stained samples were used for image analyses. Outer and inner contours of vessels at 100-times magnification in one microscopic field were measured with WinROOF program Ver.5.5.0 (Mitani Corporation, Fukui, Japan). Outer contours were expressed as surface area of vessel and inner contours were expressed as surface area of lumen.

Statistical analyses. Data are expressed as mean \pm SD. Statistical analysis was performed using the StatView J-4.5 program (Abacus Concepts Inc., Berkeley, CA). The mean 
A

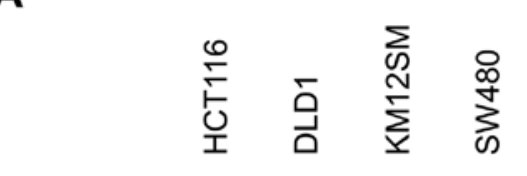

Ang2

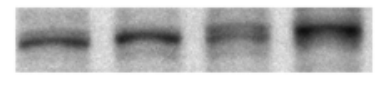

actin

B

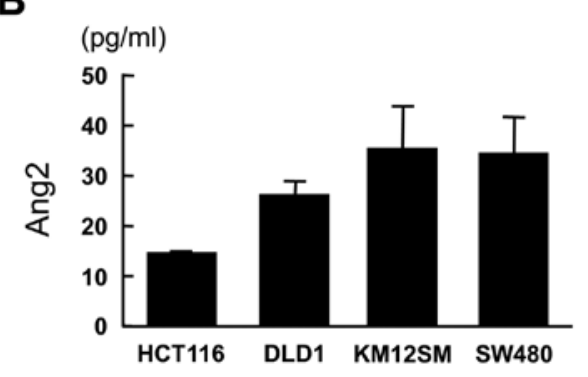

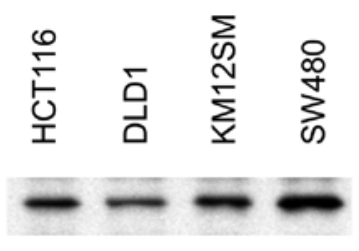

actin

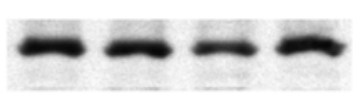

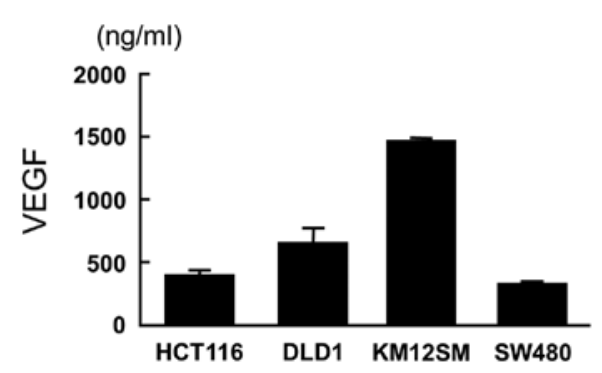

Figure 1. (A) Expression of Ang2 (83 kDa) and VEGF (42 kDa) in colon cancer cell lines. The colon cancer cell lines displayed intense expression of Ang2 and VEGF. Actin bands (42 kDa) served as a loading control of equal amount of the protein lysate. (B) Measurement of Ang2 and VEGF levels in the cultured medium. ELISA showed various levels of Ang2 and VEGF in the culture medium of each colon cancer cell line. KM12SM was found to release high level of both Ang2 and VEGF into the medium. Data are expressed as mean \pm SD from triplicate cultures.

tumour volume of each treatment group was compared by Student's t-test. A p-value $<0.05$ was considered statistically significant.

\section{Results}

Ang2 and VEGF expression in colon cancer cells. Western blot analysis showed that HCT116, DLD1, KM12SM and SW480 colon cancer cell lines exclusively displayed intense expression of both Ang2 and VEGF (Fig. 1A). ELISA showed various levels of Ang2 and VEGF in the culture medium and KM12SM was found to release high level of both Ang2 and VEGF (Fig. 1B).

Inhibition of binding affinity between Ang2 and Tie2 by L1-10 peptibody. In vitro immunoreaction test indicated that the L1-10 peptibody (L1-10) blocked the binding of Ang2 and Tie2 at more than $0.1 \mathrm{nM}$ in a dose-dependent manner, while addition of human IgG1 Fc at various concentrations did not affect Ang2-Tie2 binding affinity (Fig. 2A). To examine the activation status of the Tie 2 receptor, phosphorylation on Tyr992 of the Tie2 receptor was examined using the HUVEC with early exposure (15 min) by Ang2 or L1-10. Although Tie2 expression did not change, Ang2 inhibited phosphorylation of Tie2 receptor at $200 \mathrm{ng} / \mathrm{ml}$. This dephosphorylation by Ang2 was abolished with addition of L1-10 at $30 \mathrm{ng} / \mathrm{ml}$. (Fig. 2B).

Effects of VEGF, Ang2 and L1-10 on growth and tube formation of HUVECs. Addition of Ang2 or L1-10 did not enhance cell proliferation and tube formation of HUVECs (Fig. 3A and $\mathrm{B}$, upper panels). On the other hand, addition of VEGF at
$25 \mathrm{ng} / \mathrm{ml}$ enhanced growth and it resulted in increased number of tube formation of HUVECs (Fig. 3A and B, lower panels).

We then examined protein expression of Rac1 and CDC42 since these molecules are reportedly shown to be involved in process of tube formation of HUVECs $(15,16)$. As shown in Fig. 3C, VEGF enhanced expression of both the Racl and the CDC42 proteins, while Ang 2 at various concentrations did not affect the expression of the two proteins (Fig. 3C).

Ang2 inhibition by L1-10 treatment reduced tumour formation in nude mice. Subcutaneous injection of L1-10 (at $2 \mathrm{mg} / \mathrm{kg}$ or $5 \mathrm{mg} / \mathrm{kg}$ ) into different sites of KM12SM xenografts inhibited tumour formation dose-dependently (Fig. 4A). In the $5 \mathrm{mg} / \mathrm{kg}$ group, tumour formation was significantly inhibited from day 10 compared to that of control group. In the $2 \mathrm{mg} / \mathrm{kg}$ group, tumour formation was inhibited in later phase of experiment period. Histopathological examination of xenografts revealed that when compared to control IgG treatment (Fig. 4Ba), tumour vessels in L1-10 treated mice extended like a pine needle and lumen formation was scarcely noted (Fig. 4Bb). Double staining for CD31 and $\alpha$-SMA showed that vascular endothelial cells were tightly covered with pericytes in L1-10 treated group, whereas only partial recruitment of pericytes was found in the control group. In treatment with VEGF neutralizing antibody, tumour vessels decreased and endothelial cells were relatively covered with pericytes (Fig. 4Bc).

Image analysis for lumen formation. To evaluate lumen formation in each treatment, the contour of vessel or lumen was traced using the image analysis software, and the vessel area and the lumen area was estimated as described in Materials 
A

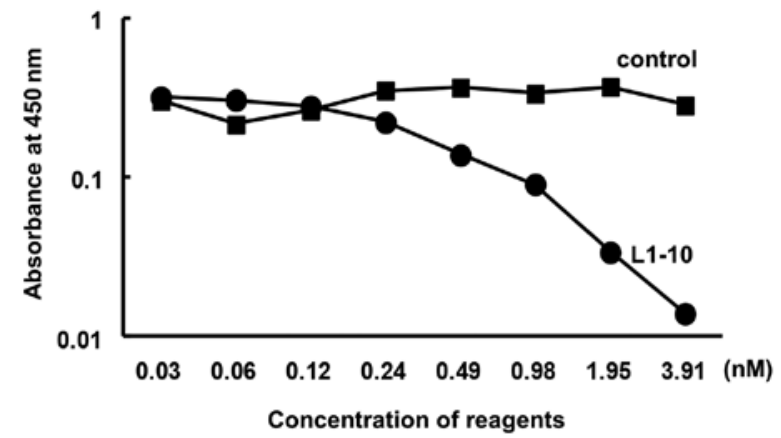

B

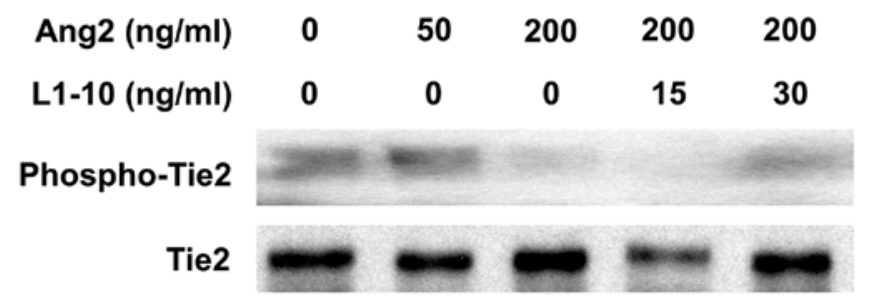

Figure 2. (A) Inhibition of binding affinity between Ang2 and Tie2 by L1-10 peptibody. Immunoreaction between recombinant human Ang2 and recombinant human Tie2 Fc Chimera was measured by tracing Tie2 amount in the Ang2-Tie2 complex in 96-well plates. L1-10 decreased binding affinity of Ang2 and Tie2 at more than $0.1 \mathrm{nM}$ dose-dependently, while addition of human IgG1 Fc at various concentrations did not affect Ang2-Tie2 binding. (B) Tie2 phosphorylation status on Tyr992 of Tie 2 was examined. HUVEC were treated by Ang2 alone (lanes 2 and 3) or Ang2 and L1-10 (lanes 4 and 5) at the indicated concentration for 15 min at $37^{\circ} \mathrm{C}$. Cell lysates were immunoprecipitated using anti-Tie2 antibody. Ang2 inhibited phosphorylation of Tie2 at $200 \mathrm{ng} / \mathrm{ml}$ and L1-10 at $30 \mathrm{ng} / \mathrm{ml} \mathrm{recovered}$ inhibition of phosphorylation by Ang2. Molecular weight of both Tie2 and phosphorylated Tie2 was $140 \mathrm{kDa}$.

and methods. Treatment with anti-VEGF antibody significantly decreased both vessel area and lumen area $(\mathrm{p}<0.01$ for both, Fig. 5A and B). On the other hand, L1-10 treatment at $5 \mathrm{mg} / \mathrm{kg}$ did not decrease vessel area (Fig. 5A), but it significantly decreased the lumen area $(\mathrm{p}<0.01$, Fig. $5 \mathrm{~B})$. When the ratio of lumen area to vessel area was calculated, there was a significant decrease in L1-10 treatment group ( $\mathrm{p}<0.01$, Fig. $5 \mathrm{C}$ ).

Combination treatment. Since the histopathological analysis indicated that inhibition of either Ang2 or VEGF showed different anti-vascular effect, we examined whether the two treatments would produce enhanced tumour inhibitory effects. After inoculation of KM12SM cells into nude mice, control IgG, anti-VEGF antibody, and L1-10 were administered as summarized in Table I.

Combination treatment with anti-VEGF and L1-10 administered from the day of inoculation significantly decreased tumour volume compared to anti-VEGF treatment except days 8 and 10 (Fig. 6A). When treatment started 5 days after inoculation, combination treatment showed no significant difference compared to anti-VEGF treatment (Fig. 6B).

\section{Discussion}

In this study, we found Ang2 inhibition exerts a superb efficacy especially in vivo. Histological analysis on xenografts planted in nude mice suggests that Ang2 inhibition could have disturbed in vivo vascular differentiation, i.e., lumen formation. There are two important aspects on tumour angiogenesis, that is, growth of vascular endothelial cells and vascular differentiation. Compared to regulation of vascular endothelial cell growth, the underlying mechanism on vascular differentiation remains largely unknown. About three decades ago, Folkman and Haudenschild observed vacuoles that penetrate from one endothelial cell to another one by in vitro system (17). Subsequent in vitro investigation gradually revealed that pinocytosis occurs via interaction of integrin-extracellular matrix through CDC42 or Rac1-dependent manner $(18,19)$. It was also demonstrated that several vacuoles, generating at 2-4 $\mathrm{h}$ in a single HUVEC, undergo intra-cellular fusion, and later at 24-48 h HUVECs make assembly body, resulting in lumen formation, raising VE cadherin as a molecular basis (20). By contrast, in vivo lumen formation has not been assessed for a long time because direct observation is rather difficult on the process of lumen formation that occurs profoundly in the animal body. In vivo tube formation of the vascular endothelial cells was reported for the first time in 2006, through observation of zebrafish by two-photon imaging system (21).

In this study, we employed L1-10 peptibody, the Ang2 selective inhibitor that showed 1,000-fold inhibitory selectivity for Ang2 over Ang1 $(7,13,14)$. We confirmed by ELISA that L1-10 abolished in vitro binding affinity between Ang2 and Tie2 in a dose-dependent manner (Fig. 2A).

We further confirmed that phosphorylation at Tyr992 of Tie 2 receptor, which had undergone dephosphorylation by stimulation with the recombinant Ang2, is rescued by the addition of L1-10. (Fig. 2B). These data indicate that L1-10 indeed blocks Ang2-Tie2 signal transduction. 
A
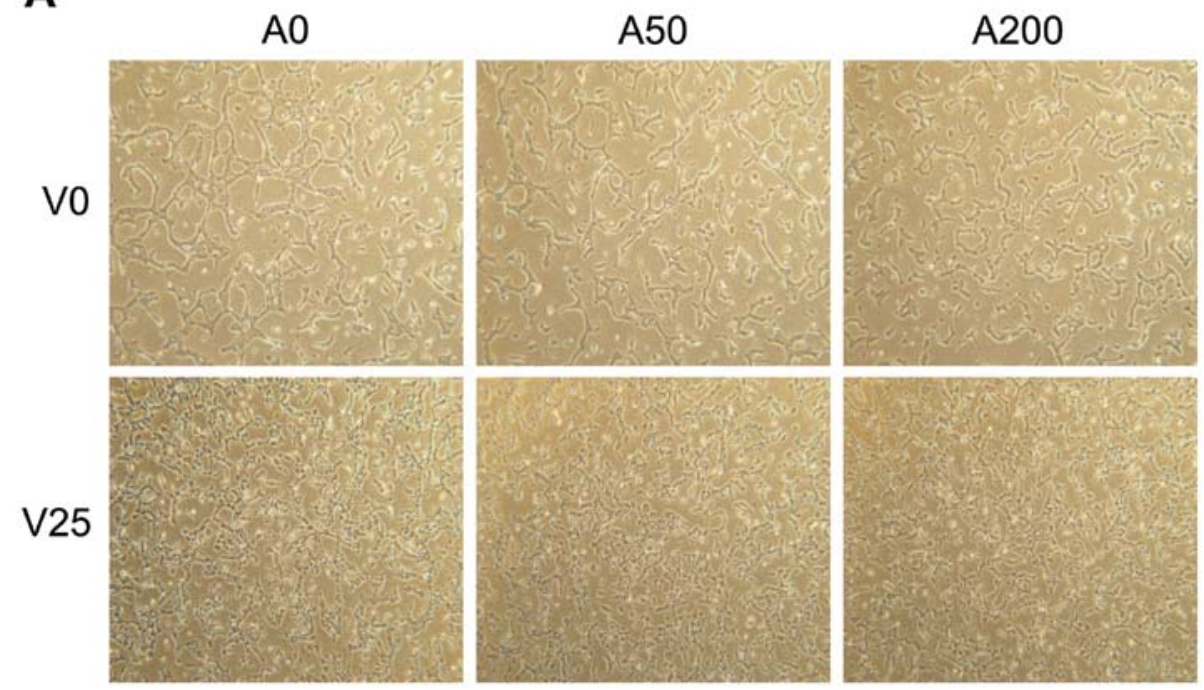

V: VEGF (ng/ml) A:Ang2 (ng/ml)

B
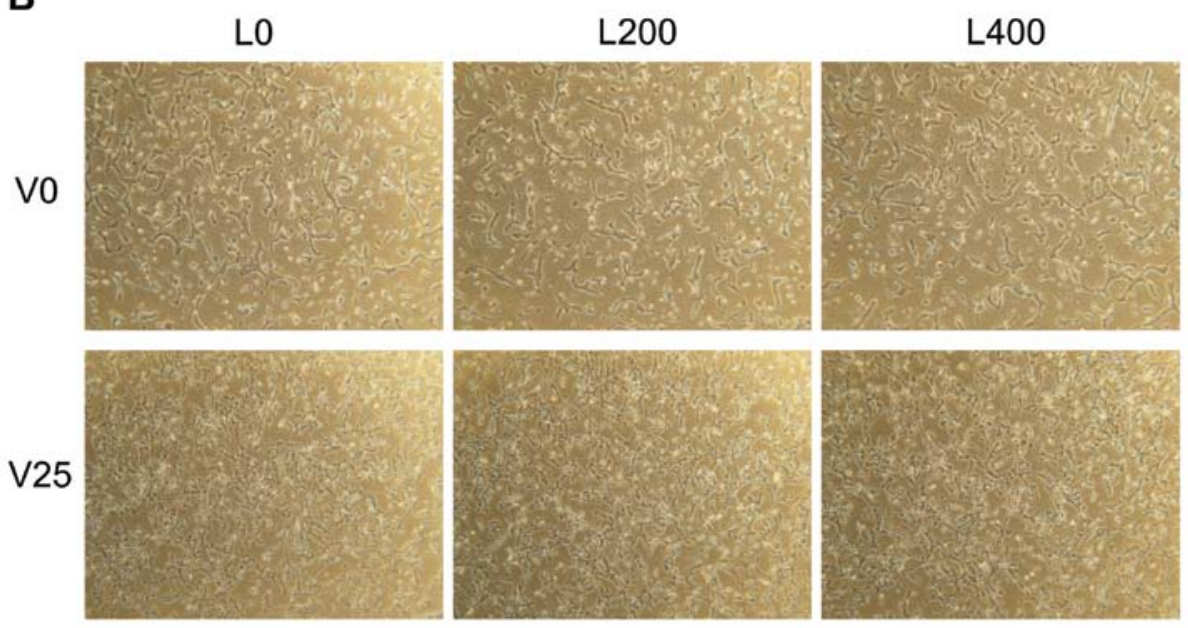

V: VEGF $(\mathrm{ng} / \mathrm{ml})$

$\mathrm{L}: \mathrm{L} 1-10(\mu \mathrm{g} / \mathrm{ml})$

C

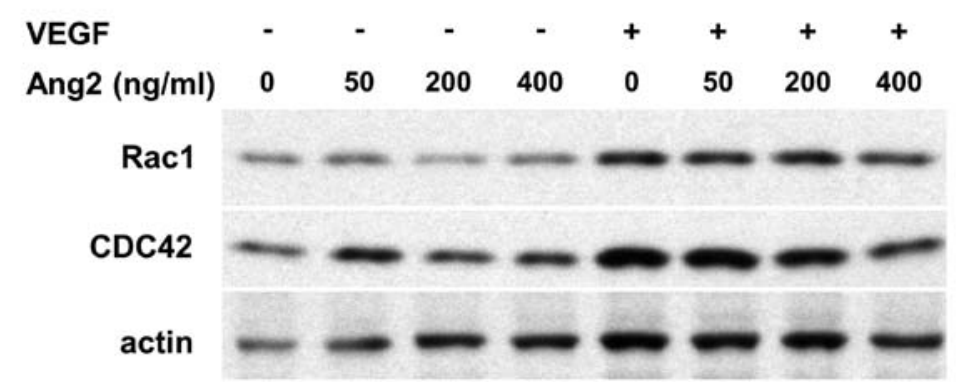

Figure 3. In vitro growth and tube formation of HUVECs. (A) Addition of Ang2 (at 50 and $200 \mathrm{ng} / \mathrm{ml}$ ) or (B) L1-10 (at $200 \mathrm{and} 400 \mu \mathrm{g} / \mathrm{ml}$ ) for $72 \mathrm{~h}$ did not affect behaviour of HUVECs when cultured in the collagen matrix, in the absence of VEGF (V0). On the other hand, addition of VEGF at 25 ng/ml (V25, lower panels) enhanced cell proliferation and resulted in increased number of tube formation of HUVECs. (C) Protein expression of Rac1 (21 kDa) and CDC42 (22 kDa) after treatment of VEGF or Ang2 for $48 \mathrm{~h}$. VEGF treatment at $25 \mathrm{ng} / \mathrm{ml}$ enhanced expression of both Rac1 and CDC42, while Ang2 at various concentrations did not affect the expression of the two proteins.

Previous reports on Ang2 inhibition in tumour cells showed decreased tumour volume. These studies used the Colo205 colon cancer cells $(7-10,22,23)$ and other colon and breast cancer cells (8), the LuCap23.1 prostatic cancer cells (13) and U-87 glioma cells (24). The KM12SM colon cancer cells employed display high levels of Ang2 and VEGF among 


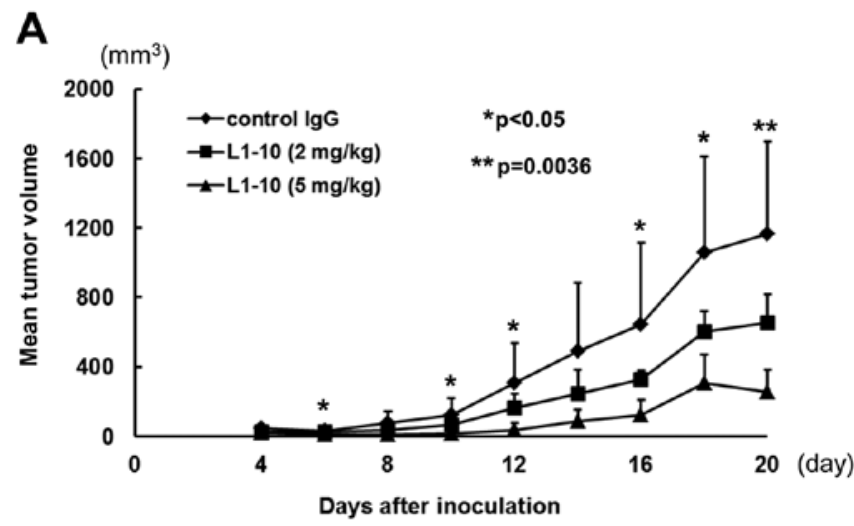

\section{$\uparrow \uparrow \uparrow \uparrow \uparrow \uparrow \uparrow \uparrow \uparrow \uparrow$}

B

(a) Control

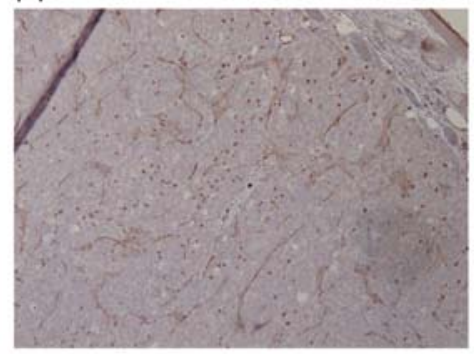

(b) L1-10 treatment

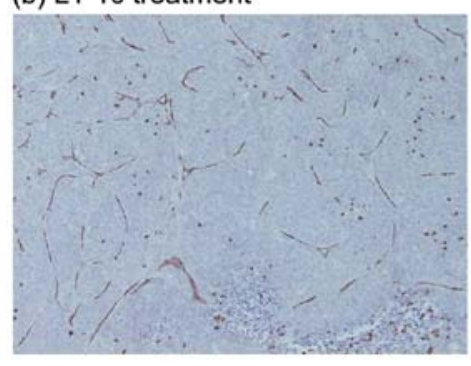

(c) Anti-VEGF antibody treatment

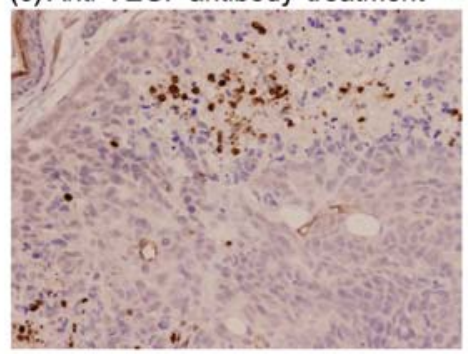

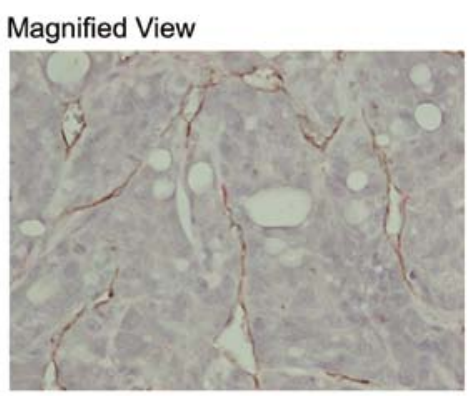
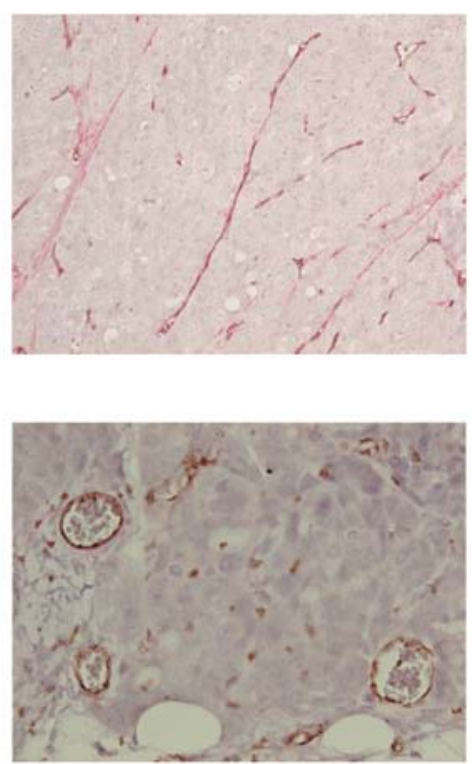

Figure 4. Growth curve of KM12SM xenograft model. (A) Subcutaneous injection of L1-10 (at 2 or $5 \mathrm{mg} / \mathrm{kg}$ ) into different sites of KM12SM xenografts inhibited tumour formation in a dose-dependent manner. (B) Histopathological examination of xenografts (a) control IgG treatment, (b) L1-10 treatment, (c) treatment with anti-VEGF neutralizing antibody. Anti-VEGF antibody was administered into peritoneal cavity at $200 \mu \mathrm{g}$ on days $0,3,6,9,12,15$ and 18 . Mice were sacrificed on day 20. Double staining for CD31 (brown) and $\alpha$-SMA (pink) was performed to label vascular endothelial cells and vascular pericytes, respectively. Magnifications (left panel, right panel): (a) x100, x400; (b) x100, x200; and (c) x200, x400.

several CRC cells (Fig. 1) and produced abundant tumour vessels in xenografts (Fig. 4). Moreover, the KM12SM cells were initially isolated as highly metastatic cells that develop marked spontaneous metastasis to liver (11) and shown to be highly activated state in $\beta$ catenin/TCF oncogenic pathway $(25,26)$. We considered that this cell type with such aggressive features could be a suitable material in evaluation of superb efficacy of Ang2-targeted therapy.

In vitro studies showed that VEGF enhanced proliferation and tube formation of HUVECs, and caused a clear increase in Rac1 and CDC42 expression when cultured in the collagen matrix, whereas neither Ang2 nor L1-10 affected in vitro 
A

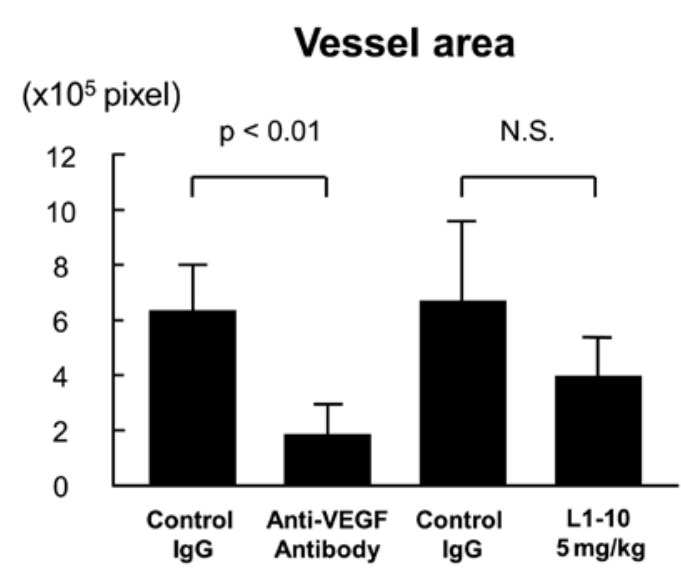

B

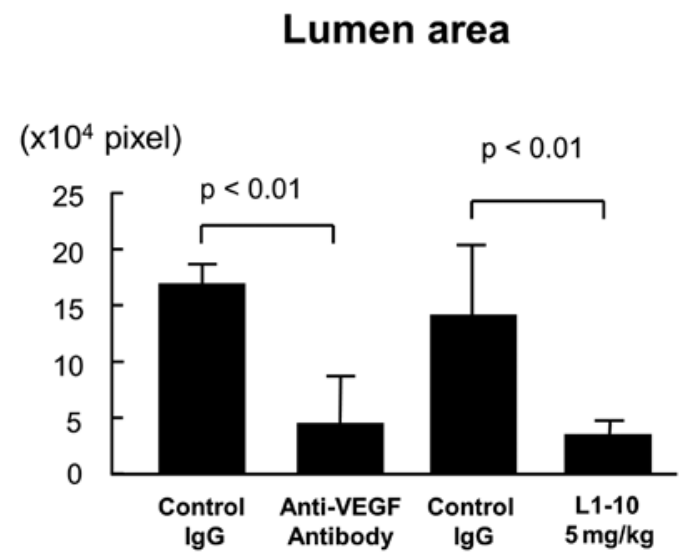

C

\section{Ratio of lumen area to vessel area}

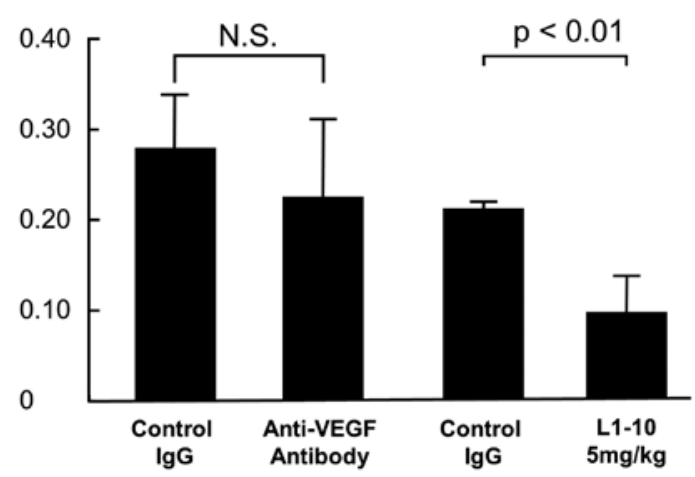

Figure 5. Image analysis for lumen formation. The outer contour of vessel or lumen was traced using the image analysis software, and the (A) vessel area, (B) lumen area, and (C) the ratio of lumen to vessel was estimated. The number of mice; anti-VEGF antibody $(n=5)$ and the control group $(n=3)$, L1-10 $(n=5)$ and the control group $(n=3)$.

behaviour of HUVECs or levels of the proteins (Fig. 3). By in vivo system we found that Ang2 inhibition with treatment of L1-10 dose dependently decreased tumour growth. Furthermore, we found that L1-10 led to extension of the tumour-associated vessels whilst it suppressed formation
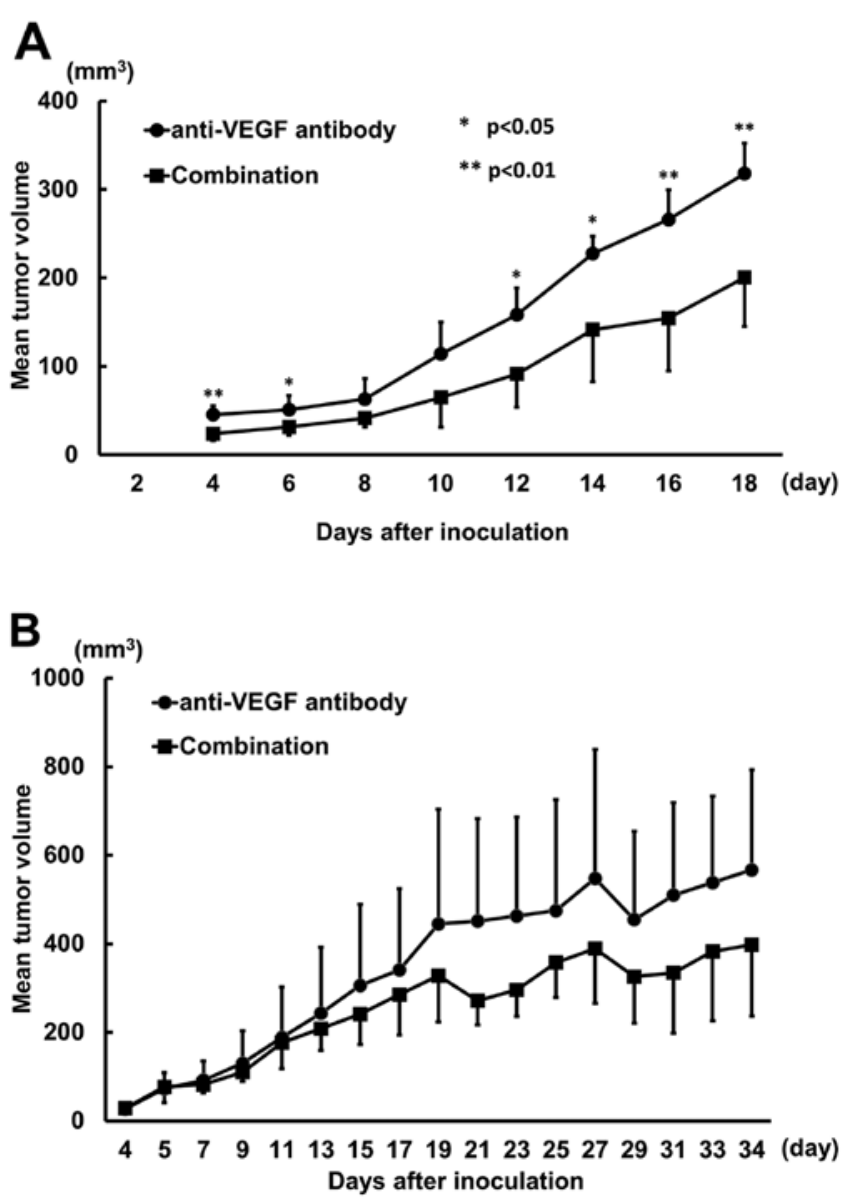

Figure 6. Growth curve of combination treatment. L1-10, anti-VEGF antibody, control IgG were administered along with routes, intervals and doses summarized in Table I. Combination treatment with early administration: injection of L1-10 and anti-VEGF antibody were started immediately after inoculation. Treatment continued to day 18. Mice were sacrificed at day 20. Combination treatment inhibited tumour growth. The decrease in tumour volume was statistically significant except for days $8(\mathrm{p}=0.0895)$ and $10(\mathrm{p}=0.0558)$ by t-test. ${ }^{*}$ and ${ }^{* *}$ represent p-value $<0.05$ and $<0.01$, respectively. Combination treatment with late administration: injection of L1-10 and anti-VEGF antibody were started 5 days after inoculation. Treatment was continued to day 31 . Mice were sacrificed at day 34 . No significant differences were observed between VEGF treatment and combination treatment in any time-point.

of a sound lumen. Ratio of lumen area to vessel area was significantly decreased by L1-10 treatment compared to that of VEGF. The double staining of both endothelial cells and pericytes revealed that the endothelial cells were tightly covered with abundant pericytes in the tumour-associated vessels of L1-10-treated mice, when compared to control groups (Fig. 4B). Therefore, the difference in effects endowed by L1-10 could be the existence of both endothelial cells and pericytes in vivo, but not in vitro in which pericytes are lacking. Our data suggest that Ang1/Ang2 balance plays an essential role in in vivo vascular differentiation. Thus, it is assumed that Ang1/Ang2 balance may be shifted to Ang1 dominance by L1-10, which should facilitate recruitment of pericytes along the endothelial cells, considering established model on angiogenesis by Angs-Tie 2 signaling (1,27). Indeed, several recent studies provided evidence that Ang 2 inhibition causes an increase in pericyte-coverage over the endothelial cells $(9,10,22)$. 
We postulate that lack of pericytes in the in vitro system unables the demonstration of the relevance of Ang2 in vascular differentiation.

Several agents against Ang2, Ab536 (7) or anti-Ang2 monoclonal antibody 3.19 .3 (8), or peptide antibody fusions including 2xCon4, L1-7, L1-10, CovX-Bodies $(7,9,10,13,22,23)$ generally suppressed in vivo tumour growth implanted in nude mice. These studies provided histological features of the tumours such as deceased vessel density, increased apoptosis and pericyte coverage. We first report here that vascular endothelial cells were extended, but lacked lumen formation by L1-10 mediated Ang2 inhibition. One may suppose that such characterized phenotype is rather specific to the KM12SM cells. However, we consider that our finding is not unique to just the cell type because Chae et al reported that forced expression of Ang2 caused enlargement of vascular lumen in xenografts of U87 glioma cells (24), in which coverage of the endothelial cells with pericytes was rather diminished by overexpression of Ang2. Therefore, it is likely that in vivo vascular differentiation is highly dependent on endothelial cell-pericyte interaction through Ang2 mediated mechanism.

We found that combination of the anti-VEGF antibody and L1-10 enhanced tumour inhibitory effects as compared to anti-VEGF alone in nude mice. This is consistent with a recent report by Hashizume et al (22). Since histological analysis revealed that anti-VEGF or anti-Ang2 treatment caused a distinct inhibitory effect on formation of tumour-associated vessels, it is probable that the suppressive effects on KM12SM tumour could be through a tumour angiogenesis-mediated mechanism. VEGF inhibition is already clinically feasible when applied with chemotherapy including 5-FU/leucovorin, oxaliplatin and irinotecan. One of its mechanisms is thought to be normalization of tumour-associated vessels which facilitate the entry of chemo-agents to the tumour cells (28). With a view of pericyte-endothelial cell interaction, Ang2 inhibition would contribute to intense coverage of vascular endothelial cells with pericytes and this is in the line with anti-VEGF for vascular normalization in tumour tissues. In conclusion, we propose that Ang2 is essential to in vivo vascular differentiation.

\section{References}

1. Bergers G and Benjamin LE: Tumorigenesis and the angiogenic switch. Nat Rev Cancer 3: 401-410, 2003.

2. Kabbinavar F, Hurwitz HI, Fehrenbacher L, et al: Phase II, randomized trial comparing bevacizumab plus fluorouracil (FU)/leucovorin (LV) with FU/LV alone in patients with metastatic colorectal cancer. J Clin Oncol 21: 60-65, 2003.

3. Ogawa M, Yamamoto H, Nagano H, et al: Hepatic expression of ANG2 RNA in metastatic colorectal cancer. Hepatology 39: 528-539, 2004

4. Hurwitz H, Fehrenbacher L, Novotny W, et al: Bevacizumab plus irinotecan, fluorouracil, and leucovorin for metastatic colorectal cancer. N Engl J Med 350: 2335-2342, 2004.

5. Maisonpierre PC, Suri C, Jones PF, et al: Angiopoietin-2, a natural antagonist for Tie2 that disrupts in vivo angiogenesis. Science 277: 55-60, 1997.

6. Suri C, Jones PF, Patan S, et al: Requisite role of angiopoietin-1, a ligand for the TIE2 receptor, during embryonic angiogenesis. Cell 87: 1171-1180, 1996.

7. Oliner J, Min H, Leal J, et al: Suppression of angiogenesis and tumor growth by selective inhibition of angiopoietin-2. Cancer Cell 6: 507-516, 2004.
8. Brown JL, Cao ZA, Pinzon-Ortiz M, et al: A human monoclonal anti-ANG2 antibody leads to broad antitumor activity in combination with VEGF inhibitors and chemotherapy agents in preclinical models. Mol Cancer Ther 9: 145-156, 2010.

9. Coxon A, Bready J, Min H, et al: Context-dependent role of angiopoietin-1 inhibition in the suppression of angiogenesis and tumor growth: implications for AMG 386, an angiopoietin1/2-neutralizing peptibody. Mol Cancer Ther 9: 2641-2651, 2010.

10. Falcon BL, Hashizume H, Koumoutsakos P, et al: Contrasting actions of selective inhibitors of angiopoietin-1 and angiopoietin-2 on the normalization of tumor blood vessels. Am J Pathol 175: 2159-2170, 2009.

11. Morikawa K, Walker SM, Nakajima M, Pathak S, Jessup JM and Fidler IJ: Influence of organ environment on the growth, selection, and metastasis of human colon carcinoma cells in nude mice. Cancer Res 48: 6863-6871, 1988.

12. Yamamoto H, Kondo M, Nakamori S, et al: JTE-522, a cyclooxygenase- 2 inhibitor, is an effective chemopreventive agent against rat experimental liver fibrosis1. Gastroenterology 125: 556-571, 2003.

13. Morrissey C, Dowell A, Koreckij TD, et al: Inhibition of angiopoietin-2 in LuCaP 23.1 prostate cancer tumors decreases tumor growth and viability. Prostate 70: 1799-1808, 2010.

14. Tressel SL, Kim H, Ni CW, et al: Angiopoietin-2 stimulates blood flow recovery after femoral artery occlusion by inducing inflammation and arteriogenesis. Arterioscler Thromb Vasc Biol 28: 1989-1995, 2008.

15. Bayless KJ and Davis GE: The Cdc42 and Racl GTPases are required for capillary lumen formation in three-dimensional extracellular matrices. J Cell Sci 115: 1123-1136, 2002.

16. Davis GE, Bayless KJ and Mavila A: Molecular basis of endothelial cell morphogenesis in three-dimensional extracellular matrices. Anat Rec 268: 252-275, 2002.

17. Folkman J and Haudenschild C: Angiogenesis in vitro. Nature 288: 551-556, 1980.

18. Bayless KJ, Salazar R and Davis GE: RGD-dependent vacuolation and lumen formation observed during endothelial cell morphogenesis in three-dimensional fibrin matrices involves the alpha(v)beta(3) and alpha(5)beta(1) integrins. Am J Pathol 156: 1673-1683, 2000.

19. Davis GE and Bayless KJ: An integrin and Rho GTPasedependent pinocytic vacuole mechanism controls capillary lumen formation in collagen and fibrin matrices. Microcirculation 10: 27-44, 2003.

20. Yang S, Graham J, Kahn JW, Schwartz EA and Gerritsen ME: Functional roles for PECAM-1 (CD31) and VE-cadherin (CD144) in tube assembly and lumen formation in threedimensional collagen gels. Am J Pathol 155: 887-895, 1999.

21. Kamei M, Saunders WB, Bayless KJ, Dye L, Davis GE and Weinstein BM: Endothelial tubes assemble from intracellular vacuoles in vivo. Nature 442: 453-456, 2006.

22. Hashizume H, Falcon BL, Kuroda T, et al: Complementary actions of inhibitors of angiopoietin-2 and VEGF on tumor angiogenesis and growth. Cancer Res 70: 2213-2223, 2010.

23. Huang H, Lai JY, Do J, et al: Specifically targeting angiopoietin-2 inhibits angiogenesis, Tie2-expressing monocyte infiltration, and tumor growth. Clin Cancer Res 17: 1001-1011, 2011.

24. Chae SS, Kamoun WS, Farrar CT, et al: Angiopoietin-2 interferes with anti-VEGFR2-induced vessel normalization and survival benefit in mice bearing gliomas. Clin Cancer Res 16: 3618-3627, 2010.

25. Herynk MH, Tsan R, Radinsky R and Gallick GE: Activation of c-Met in colorectal carcinoma cells leads to constitutive association of tyrosine-phosphorylated beta-catenin. Clin Exp Metastasis 20: 291-300, 2003.

26. Kim Y, Park H, Lim Y, et al: Decreased syndecan-2 expression correlates with trichostatin-A induced-morphological changes and reduced tumorigenic activity in colon carcinoma cells. Oncogene 22: 826-830, 2003

27. Shim WS, Ho IA and Wong PE: Angiopoietin: a TIE(d) balance in tumor angiogenesis. Mol Cancer Res 5: 655-665, 2007.

28. Jain RK: Normalization of tumor vasculature: an emerging concept in antiangiogenic therapy. Science 307: 58-62, 2005. 